\title{
The Value of Plain Radiography in Suspected Intraocular Foreign Body
}

\author{
L. C. BRAY and P. G. GRIFFITHS \\ Newcastle
}

\begin{abstract}
Summary
Patients presenting to an eye casualty department with a history of exposure to high velocity particles often undergo radiological investigation to rule out clinically undetected intraocular foreign bodies (IOFB). We reviewed the indications for and the results of $\mathrm{X}$-rays performed in our eye department over a five year period to determine the effectiveness of such screening. Our results suggest that most of the $X$-rays are performed needlessly and that radiological investigation could be restricted to those patients with clinical evidence of penetrating ocular and orbital trauma and patients wtih subconjunctival haemorrhage.
\end{abstract}

The risk of ocular penetration by high velocity foreign bodies is well recognised and a legitimate cause for concern. ${ }^{1}$ Intraocular foreign bodies (IOFBs) may be missed only to present later with complications such as endophthalmitis, retinal detachment, or irreversible damage due to metallosis. ${ }^{2}$ This fear has led to a view that all ocular injuries from high velocity particles should X-rayed.

The detection of foreign bodies (FBs) by conventional radiology is dependent on their relative density compared to water. ${ }^{3}$ Consequently though X-rays will often reveal metallic FBs, the detection of glass, perspex and wood is much less reliable. Notwithstanding these limitations and despite the absence of data to support their use as a screening test, it is standard clinical practice to screen patients with ocular injuries from high velocity particles with orbital X-rays in many ophthalmic and casualty departments.

We reviewed the results of radiological investigation carried out on a group of such patients to evaluate its contribution to clinical management and to determine its effectiveness as a screening procedure.

\section{Materials and Methods}

All patients who underwent orbital radiography for suspected IOFB between January 1986 and December 1990 were identified from the Newcastle General Hospital eye casualty department records. Details of their history, clinical examination, and the $\mathrm{X}$-ray investigation (reported by a member of the radiology department in each case) were retrieved from their clinical records and recorded on a data base for subsequent analysis. All patients were initially examined by an ophthalmologist then subjected to radiographic investigation comprising modified occipitomental (with chin elevated $35^{\circ}$ ) and lateral views with exposure for soft tissues.

The patients were divided into four groups as follows: 
Group 1: Patients with penetrating ocular trauma

Group 2: Patients with periocular trauma and an intact globe

Group 3: Patients with no clinical sign of ocular or orbital penetration

Group 4: Patients whose clinical records were not retrieved

The results of these groups were analysed separately.

\section{Results}

During the above period (1986-1990) 1137 patients underwent orbital $x$ ray for suspected IOFB. The clinical records of 1019 of these were retrieved including all those who became registered as hospital in- or outpatients. The remaining 118 patients were treated solely in the casualty department. Though their casualty records were not found, the $x$ ray report was retrieved in all cases. The $\mathrm{x}$-ray results of the four groups are summarised in Table I.

\section{Group one. Patients with penetrating ocular trauma}

There were 64 patients in this group, of whom 34 had IOFBs and three had intraorbital FBs. Presenting histories of these patients are shown in Table II. The majority of patients with IOFBs presented with a history of exposure to a high velocity particle in contrast to those with uncomplicated penetrating injury most of whom received a direct blow to the eye (by objects such as bolts and screwdrivers). None of the patients were noted to have corneal FBs.

One patient presented with a history of gradually deteriorating vision following an accident nine months previously in which he noted an FB sensation in his right eye after using a hammer and chisel. His clinical examination by a general casualty officer and his orbital radiographs reported by a consultant radiologist were normal. Subsequent examination in the eye clinics revealed a healed full thickness corneal laceration and a visible intralenticular metallic foreign body.

Of the $37 \mathrm{FBs}, 29$ were metallic of which 26 $(90 \%)$ were visible on orbital radiographs, seven were glass of which five $(71 \%)$ were visible, and one which was wood was not visible on X-ray. All films were considered to be of adequate technical quality. Of the six IOFBs undetected by radiography, four were visible on clinical examination and two (one wood and one glass) were found during surgical repair of the ocular penetration.

\section{Group two. Patients with periocular trauma}

There were 75 patients in this group, 69 of whom had a subconjunctival haemorrhage $(\mathrm{SCH})$, a conjunctival laceration or both. There were 16 periocular FBs (comprising ten subconjunctival and six intraorbital), 13 of which were reported on orbital radiographs. All of the subconjunctival FBs (eight metallic, one glass, and one wood) were identified prior to X-ray studies. Seven of these (all metallic) were identified by radiography. Four of the intraorbital FBs were air gun pellets and two were large fragments of glass. All were associated with major periocular trauma.

\section{Group three. Patients with no evidence of ocular penetration}

There were 880 patients in this group. The common presenting histories and major findings on clinical examination (one 'finding' per patient) are shown in Table III. Of the 880 radiographs taken, five showed evidence of an FB, two of which were shown to be artefactual by repeating the X-rays. In two cases, the FB was lodged in an eyelid and incidental to the injury under investigation. The other positive $x$ ray demonstrated a corneal $\mathrm{FB}$ which had

Table I. Summary of the X-ray findings for all patients

\begin{tabular}{lcccc}
\hline & \multicolumn{3}{c}{ X-ray report } \\
\cline { 2 - 5 } & FB absent & FB present & False positive & False negative \\
\hline Group 1 & 33 & 31 & 0 & 6 \\
Group 2 & 62 & 13 & 0 & 3 \\
Group 3 & 875 & 5 & 2 & 17 \\
Group 4 & 118 & 0 & 0 & Not known \\
\hline
\end{tabular}


Table II. Presenting histories of patients with penetrating injuries in the presence and absence of $I O F B$

\begin{tabular}{lcc}
\hline History & IOFB present & IOFB absent \\
\hline Hammering & 22 & $3^{\prime}$ \\
Direct trauma & 3 & 15 \\
Flying glass & 7 & 7 \\
Power tools & 2 & 1 \\
Miscellaneous & $3^{\mathrm{B}}$ & $1^{\mathrm{C}}$ \\
\hline
\end{tabular}

$\mathrm{A}=$ by metal objects such as bolts, screwdrivers etc.

$\mathrm{B}=2$ shot by airgum, 1 welding.

$\mathrm{C}=1$ vague historian.

not been removed prior to $\mathrm{X}$-rays being taken. A further 17 patients with metallic corneal FBs had orbital $x$ rays taken prior to removal of the FB. No radio-opaque material was seen in any of these cases.

\section{Group four. Patients whose hospital record was not retrieved}

There were 118 patients in this group. All were treated solely in the casualty department, none having been registered as either hospital outpatients or inpatient. The results of orbital X-rays, which were retrieved in all cases, were universally reported as normal. It is, of course, not known how many of the $x$ rays were false negative as we have not had access to the patients clinical records.

\section{Discussion}

Plain $x$ rays identified a large proportion of FBs associated with penetrating ocular trauma and are an invaluable aid to their identification and localisation particularly in the presence of intraocular blood or media opacity. However, doubts concerning the reliability of orbital X-rays in the detection of IOFBs remain, even when the FB is metallic. A recent study ${ }^{4}$ reported an IOFB detection rate by plain X-rays of $69 \%$ for metallic materials, $77 \%$ for glass but only $0-15 \%$ for perspex, wood and graphite. Our figures for metallic $(90 \%)$ and glass FBs $(71 \%)$ broadly concur with these findings.

There was a large group of patients with $\mathrm{SCH}$ and/or conjunctival lacerations. Though all subconjunctival FBs in this group were detected clinically, and only $70 \%$ of these were confirmed by orbital $x$ ray, we would argue that such investigation is necessary as a means of detecting subconjunctival and scleral FBs obscured by $\mathrm{SCH}$, and ciliary body FBs that are undetectable by indirect ophthalmolscopy.

Turning to the group with nonpenetrating trauma (group 3), $x$ rays were almost invariably reported as normal even in the presence of a metallic corneal FB. Many of these patients were involved in activities not associated with significant risk fo IOFB such as grinding, and others presented merely with 'FB sensation'. Consequently, none of these patients require radiological investigation. Of those exposed to high velocity particles, the majority had either superficial (and by implication low velocity) FBs or epithelial abrasions which accounted for their symptoms. Clinical examination in these cases was otherwise normal and radiological investigation, consequently, unnecessary. Only two previously undetected FBs were revealed by $\mathrm{X}$-ray and, in both cases, were clinically insignificant and incidental to the injury under investigation.

Table III. Presenting histories and main clinical finding in patients without penetrating ocular or orbital trauma

\begin{tabular}{|c|c|c|c|}
\hline \multicolumn{2}{|l|}{ Presenting history } & \multicolumn{2}{|l|}{ Clinical findings } \\
\hline Hammering & 350 & $\mathrm{FB}^{\mathrm{C}}$ & 577 \\
\hline Grinding & 184 & Abrasion & 153 \\
\hline Power tools & 152 & A/C activity & 22 \\
\hline Direct trauma ${ }^{\mathrm{A}}$ & 52 & $\begin{array}{l}\text { Corneal } \\
\text { Laceration (partial thickness) }\end{array}$ & 18 \\
\hline Welding & 39 & Hyphaema & 18 \\
\hline Broken glass & 32 & NAD & 83 \\
\hline Other $^{B}$ & 71 & Other $^{\mathrm{D}}$ & 9 \\
\hline
\end{tabular}

$\mathrm{A}=$ By metal objects such as bolts, screwdrivers etc.

$\mathrm{B}=$ Mainly 'FB sensation'.

$\mathrm{C}=$ Corneal, conjunctival or subtarsal FBs.

$\mathrm{D}=$ Mainly lid oedema, 'injection'. 
The records of 118 patients were not retrieved. All were treated solely in the casualty department, and all had orbital $x$ rays reported as normal. We do not believe that the absence of these records alters or detracts from our argument as in none of these cases could the $x$ ray have contributed to the detection of an IOFB.

In no case was an IOFB detected on X-ray, without concomitant evidence of ocular penetration. Clinical examination conducted by a competent ophthalmologist is therefore the most important investigation. This should comprise slitlamp biomicroscopy of the anterior segment and binocular indirect ophthalmoscopy of the fundus following dilatation of the pupils. In the absence of evidence of ocular penetration, screening by orbital $x$ ray is inappropriate as the true positive rate is too low and the false negative rate too high for this to be effectively performed. ${ }^{5}$

Thus, patients exposed to high velocity fragments should only be subjected to orbital radiography in the presence of clinically confirmed or strongly suspected penetrating ocular or orbital trauma or SCH. In cases where junior ophthalmologists cannot confidently exclude the presence of IOFB by clinical examination, senior opinion, rather than $x$ ray investigation, should be obtained. By adopting this protocol, in excess of $80 \%$ of orbital $x$ rays in this series need not have been performed, considerably reducing both patient exposure to ionising radiation and the financial cost to the department.

Key words: intraocular foreign body, orbital X-ray.

\section{References}

${ }^{1}$ Bartkowski SB, Kurek M', Krystkowa K, Zapala J: Foreign bodies in the orbit: A review of 20 cases. J Maxillo-Facial Surg 1984, 12: 97-102.

${ }^{2}$ Duke Elder S: System of Ophthalmology. St Louis, CV Mosby Co 1972; vol 14: pp 508-543, 560-564.

${ }^{3}$ De Lacey G, Evans R, Sandin B: Penetrating injuries: how easy is it to see glass (and plastic) on radiographs? Br J Radiol 1985, 58: 27-30.

${ }^{4}$ Bryden FM, Pyott AA, Bailey M, McGhee CNJ: Real time ultrasound in the assessment of intraocular foreign body. Eye 1990, 4: 727-731.

${ }^{5}$ Roberts CJ: Epidemiology for Clinicians. Tunbridge Wells. Pitman Medical: 1977, 86-89. 